\title{
Integrated Chinese Poetry Therapy with Rhythm as the Core
}

\section{《以节奏为核心的中国整合性诗歌治疗》}

\author{
Peng Yongwen 彭勇文 \\ Shanghai Theatre Academy, China
}

\begin{abstract}
China is a country of poetry with a history of more than 3,000 years. From the perspective of creative art therapy, poetry, like music, dance, and drama, is a good way to help people release and balance their emotions, find meaning, and transform their life. In this article, the author explores Chinese-integrated poetry therapy with rhythm as the core, integrating the breathing and movement training of tai chi chuan and the voice and language training of recitation art. In his practice, he uses Chinese classical verses or prose writings with different characteristics of yin and yang, so that people can draw positive psychological energy from them and maintain their physical and mental health.
\end{abstract}

Keywords: rhythm, integrated poetry therapy, tai chi chuan, poetry recitation, physical and mental health

\section{摘要}

中国是一个诗歌的国度，有超过三干多年的传统。从创造性艺术治疗的角度看，诗歌 与音乐、舞蹈、戏剧一样，都是帮助人们平衡情绪、发现意义、转化生命的良好方 式。笔者在本文所探讨的中国整合性诗歌治疗，以节奏为核心，融合了太极拳的呼吸 和动作训练，以及朗诵艺术的声音和语言训练，合理运用不同特性的诗歌韵文或散 文，刚柔并济、阴阳调和，让人从经典文本中汲取积极的心理能量，从而促进个人或 团体的身心健康。

关键词: 节奏，整合性诗歌治疗，太极拳，诗歌朗诵，身心健康

Rhythm is the key factor in natural and biotic movements, emotional fluctuations, and artistic creations and appreciations. This article discusses the practice of rhythm training for the promotion of people's physical and mental health and the role of and/or contribution of Chinese culture to this practice. It focuses on three aspects of rhythm training. The first is the meaning of rhythm. The second is the value of rhythm training. The third is the focus on the two ways of rhythm training: training of tai chi chuan breathing and movement and training of voice and poetry recitation. 


\section{The Meaning of Rhythm}

The narrow meaning of rhythm refers to the rhythm of various literary works - especially music. According to the Encyclopedia of China: Volumes of music and dance (p. 314, 1989), "rhythm is one of the three major elements of music along with the tune and harmony. It is the organized order of the tone. Strong and weak, fast and slow, tight and loose are the key elements of rhythm which help organize the music into a unified whole to express the feelings and ideas of musician."

Rhythm is not only important for music and dance, but for almost all arts, since they all need phrasing and a primary movement pattern, and as such, is an organizing way to understand and make meaning of the world. We rely on the movement patterns to communicate. As Lin (p.249, 2012), one of the greatest modern litterateur of China, said in the Biography of Su Dongpo, "All the problems in art are rhythm problems. Whether it is painting, carving or music each art form has an implicit rhythm...."

In a broad sense, rhythm "is the organizing principle of all movements, the regularity of the various parts of each other to form a whole." (Guriyev, p.17, 1983) Everything moving in time and space shows a certain rhythm, and rhythm creates a perceivable pattern, whether stable and regular or erratic and unstable, thereby completing a certain function. Any rhythm contains two basic dimensions of speed and strength: speed, which is the fast or slow status of the movement of various parts of the object in the time dimension, and strength, which is the strong or weak status of energy. Only at the right speed and strength can the movements of the various parts can work together to form a unified rhythm and become a whole.

Sunrise and sunset, cold and hot seasons, they are grand rhythms; countless lives on earth arrange their activities and spend lifetimes according to this rhythm. The breathing of the human body, the beating of the heart, and even the opening and closing of the eyelids, they are subtle rhythms. When the rhythm of various systems and organs of the human body runs stably and evenly, people are in a healthy state; when the rhythm appears to be fluctuating or disordered, people will become sick or even die.

\section{The Significance of Rhythm Training to Promote Physical and Mental Health}

A good rhythm allows people to achieve their intended purpose with the least amount of effort to get the most out of an activity and to feel the joy of such rhythm. One example is the porter who transports cargo - the rhythm of the undulating movement and keeping pace with the speed of walking allow a porter to save effort and go further.

Another example is learning how to drive. When people are still learning to drive, they are often in a hurry and careless. After repeated practice, however, they find the rhythm of their hands and feet and thus drive more smoothly. They all engage in universal patterns of movement (regardless of the task), and each has a distinct rhythm that once recognized and embodied, allows the movement to unfold with ease. Zhuangzi, one of the great Daoism philosophers 2,000 years ago, has already revealed this. In the fable Zhuangzi: Healing Master, (Zhang, p.47, 1991) there are several interesting paragraphs such as "The king's cook cutting an ox," which is about a cook cutting up an ox for King 
Wen Hui. Whenever his hand touched, his shoulder leaned, his foot trod, and his knee thrust, there were sounds of ripping and slicing, which kept time with the rhythm of the dance of Mulberry Grove and were as melodious as the music of Jingshou. "Ah! Very good!," Wen Hui said, "How did you achieve such perfection in your skill?" The cook said that his knife has been in use for nineteen years and has cut up several thousand oxen, and yet its edge is still sharp as if it were newly whetted. Why? It was because he got the right rhythm to do his work. King Wen Hui also learned the way of nurturing life from seeing the cook's work and hearing his words.

Zhuangzi believes that human society is full of intricate contradictions and many difficulties. If we want to protect ourselves, we should keep on practicing our skills of dealing with different things and communicating with different persons. After we get the right rhythm to live in the world, we will be as satisfied and comfortable as the cook in the fable. This is the wisdom of Chinese culture; in China's traditional philosophy and theories of health, people care about the rhythm of life, the way of golden mean, the balance of Yin and Yang. China's economy and society have developed rapidly for nearly 40 years; nowadays, more and more emphasis is given to the pace and quality of development, but fewer people pay attention to the harmony between man and nature. Individuals, after intense and efficient work or study, should also actively slow down, calm down, restore a soft and natural state, and let life be nourished, so as to accommodate all things and achieve harmonious symbiosis.

\section{Two Methods of Rhythm Training to Promote Physical and Mental Health}

With the practice of rhythm training to promote physical and mental health, the author mainly adopts two methods: tai chi chuan and poetry reading. Tai chi chuan can maintain health and fitness and let the body really relax and settle down in the flowing way of breathing and movement. Voice training and poetry reading aloud can be pleasing to the heart - with the resonance of the voice and the rhythm of the language allowing us to appreciate the power of the human spirit and the unique beauty of the language, so that the inner emotions and positive energy are stimulated.

\section{Tai Chi, Body Poetry}

As one of the treasures of Chinese traditional culture, tai chi chuan is a comprehensive movement of both body and mind. It is full of both the dynamic and the static - it is softly beautiful. It can be described as "physical poetry," which is beneficial to the body's self-perception and rhythm training. Accompanied by soft and quiet Guqin instrument, the author led group workshop members in practicing the basic movements of Chen's tai chi chuan's "infinite stance," "opening and closing stance," and "wrapped stance" and cooperated with breathing and mind retracting in order to help everyone enter a stable, peaceful rhythm.

"Infinite stance" focuses on the natural looseness of the body and the feeling of staying in the dantian (lower abdomen), thus enhancing the body's sense of center of gravity and stability. Regular practice allows us to develop good strength, gain inner stability and self-confidence, and alleviate anxiety. Breathing is one of the most basic 
rhythms of the human body. The "opening and closing stance" exercises the opening and closing of the arms, the lifting and lowering of the body, and the release of the mind, so that the students can actively control the in and out movement of the breath, enhance the breath and breathing, and focus on the internal and external rhythms. To make the inner spirit fuller, practicing within the environment of parks, green spaces, etc. allows us to become more integrated with nature. "Wrapped stance" is similar to the "cloud hands" in Beijing opera. The practitioner's body center of gravity moves smoothly and slowly to the left and right, and the two arms are driven by the waist as the axis, alternating up and down. If this is practiced often, not only will the heart become quiet and focused with the continuous and recurring rhythmic movement, blood flow will become comfortable and warm, and the body will become more sensitive to the surrounding space, thus forming a good environment with association and interaction. On the basis of these three basic stances, a practitioner can continue to learn the routines or push hands of tai chi.

Tai chi is one of the most popular sports in China. After long-term training, many people not only become capable of performing tai chi chuan routines, but they also develop generous and graceful movement as well as rhythm. In their daily life, their speech and behavior often reveal a calm demeanor momentum demeanor. This develops into a warm and intimate way for people to live. This is precisely because tai chi chuan is an integrated movement with body, mind, and breath and emphasizes both internal and external exercises; practicing movement and enlightenment must be carried out simultaneously in order to smooth out the rhythm of one's life and finally achieve the effect of both physical and mental unity.

\section{Poetry, Tai Chi of the Mind}

Poetry allows poets to openly express their sincere and strong emotions and readers to truly experience and understand themselves. In such a meeting with the hearts of ancient and modern Chinese and foreign poets, readers stimulate emotional resonance, release certain negative emotions, and enhance other emotions that promote balance.

The content of the poem is lyric speech; the word of poetry should not only possess the aesthetic sense of hearing - and phonology and rhythm of poetry needs to be meticulously organized - but it also needs to have a visual aesthetic. The overall image, the picture, and the artistic conception all need to be cleverly arranged. Therefore, in order to make full use of the role of poetry in mental health and spiritual growth - and before the beginning of a poetry reading — we need adjust both the rhythm of body and breathing. Performing specific "voice tuning" means to complete basic sound training; to find the support of the breath, the articulation of the tongue, and the resonance point of the voice; and to let the sound be connected with inner feelings. Breathing and the mind should be introduced into the abdomen while the innate energy source of the body should be stimulated and applied to promoting health and balance of both mind and body. Chinese people call the lower part of the abdomen "dantian," which in Chinese traditional medical theory, is directly related to the physical and emotional health of a person. 
When a person's body, breathing, and voice are adjusted to a better status, much like when the strings of an instrument are calibrated, they can read aloud some selected poems and feel the beautiful rhythm and mood such as the poem Viewing the Waterfall at Mount Lu (p.65, 2007, Translated by Xu Yuanchong), written by one of the greatest poets during the Tang dynasty, Li Bai:

Sunlight streaming on Incense Mount kindles a violet smoke,

Far off I watch the waterfall plunge to the long river,

Flying waters descending straight three thousand feet,

Till I think the Milky Way has tumbled from the ninth height of Heaven.

Reading this poem aloud always helps participants experience grandeur. The beautiful scenery of the mountains and waterfalls and the magnificent momentum of the waterfalls always mobilize the imagination of the people. Through the sound resonance from low to high, and the corresponding expressions and body movements, they are often deeply impressed, become open-minded, imagines flying.

$\mathrm{Xu}$ Zhimo, one of the greatest modern Chinese poets, wrote the poem (p.48,1983) "On Hearing the Chant of Intercession at Tian Ning Temple at Changchou" in 1930s, which is best for people to read with low voice because it is full of various moving images and sounds and rich rhythm changes. People can experience four major rhythms as the following:

Fast and light:

Like hearing, among Himalayan peaks, echoing through innumerable ravines of shining snow the rush of clouds from beyond the sky driven by winds from beyond the sky

Fast and heavy:

Like hearing, on some ocean rock savagely struck by breakers like fierce tiger.

Slow and light:

Like hearing, through the tropic air eiderdown-soft of a desert night when the moonlight's tender fingertips stroke lightly one by one the scorched fragments of rock

Slow and heavy:

Like hearing, in a sequestered valley of the hills where the bold stars of dusk alone illuminate a world bereft of sunlight and the grasses and the trees are bowed in silent prayer.

In practice, the students use the rhythm of poetry to drive their external body movements and inner emotional activities according to the author's method. This kind of experience is unprecedented for many people, but they benefit deeply from such experiences. Poetry has a strong sense of power, and people are influenced when they read and taste these poems - they are stimulating, triggering greater emotional resonance, enlightenment, and more understanding of the sense of existence and meaning of life, thus generating 
the role of motivation or healing. The tradition of poetry in China has started more than 3,000 ago and has enchanted countless literati to find a spiritual home for themselves. For today's Chinese, poetry, prose, and other literary works, through their vocal reciting, taste, and resonance, not only can restore the sensibility and spirituality of the human heart, but also help one appreciate and understand more beautiful things in the world. This work can help to cultivate a peaceful and stable psychological quality that can withstand the unpredictable and sometimes unavoidable distress in the human world, so that people are not confined to the abyss of tragic feelings.

\section{Integrated Poetry Therapy}

The Chinese philosopher Lao Tzu (p.77, 2008, Translated by Ju Yanan) said in the 77th chapter of the Dao De Jing, "Is the Way of Heaven Not Like the Bending of a Bow?, "When it is too high, pull it down. When it is too low, push it up. He who has too much gets cut. He who has not enough gets help. The Way of Heaven is to cut excess and help one who doesn't have enough." It is the balance and adjustment of the rhythm of life.

In the education of people's physical and mental health, we can use different styles of poetry to help people adjust the balance of yin and yang of their emotions. For those with lower physical and mental energy states (such as those with depression or those who feel numb, tired, and even pessimistic about life and work) we can "push it up," and for people who are often anxious and nervous, we can "pull it down." It is the integrated therapy that uses tai chi, voice, and poetry.

Whether it is the high, the low, the rest, or the deficiencies, we can use them to practice the physical and respiratory movements of tai chi in order to make the body more peaceful, softer, loose, and warm. We can then practice the abdominal sound (or dantian's core practice of vocalization), which will help them feel the resonance of their bodies and minds, their mutual resonance, and enhance interpersonal connections.

If we want to "push it up," we can use reading and even singing loudly so that our breathing capacity increases and the flow of energy in both body and mind becomes more active. What we use is mainly masculine, unconstrained poetry, mobilizing our will and inspiring our inner strength. The theory of ancient Chinese poetry has always emphasized the fundamental driving force of human beings, the need for self-realization and selffulfillment, and the arduous and detached state of mind in the face of the hardships and difficulties of life. Among the ancient Chinese poetry, there are a large number of such poems - such as Invitation to Wine written by the great poet Li Bai of the Tang Dynasty.

If we want to "pull it down" and be peaceful, we use a small voice, whispering, to make breathing more stable and even and the flow of energy more moderate. What we use is mainly feminine, graceful poetry, leading us through the sound and into the wonderful, long-range, quiet mood of poetry; this is just like listening to all kinds of soothing light music. Poetry has more specific and much clearer images than music. Moreover, Chinese language has rich changes in pronunciation, phonology, and rhythm. Ordinary people are more likely to feel the music in poetry in the whispering. Sensual beauty - and the use of words to evoke imagination - allow us to obtain the inner visual beauty that helps open the mind and vision and connect with the greater existence, 
overcoming excitement and anxiety and making ourselves smoother and calmer. There are many poetic treasure houses in China such as the Tang Dynasty poet Chen Ziang's "Deng Youzhou Taiwanese Songs," the Song Dynasty poet Li Qingzhao's "Southern Songs," and "Luo Meng Ling," and the modern poet Xu Zhimo's "Farewell to Cambridge" and his other poems.

\section{Conclusion}

Rhythm training is beneficial not only to people's daily expressions, but also to people's self-cultivation - creating a clean and peaceful physical and mental status. If we find good rhythm in our work and life, we will have more happiness. For social organizations, only when the roles of the people are clear and they work together around the common goal with good rhythm can they achieve long lasting development.

\section{About the Author}

Dr. Yongwen Peng is an associate professor in Shanghai Theatre Academy. He has a $\mathrm{PhD}$ in performance studies, and his work focuses on arts education and therapy. His book Theatre and Training (2011) is the first of its kind in China, and he has translated The Couch and the Stage: Integrating Words and Action in Psychotherapy (2012), a book on drama therapy by Professor Robert Landy of New York University. He is a board member of Chinese Arts Therapist Group and a member of the North American Drama Therapy Association and National Association for Poetry Therapy. He is also a voice artist and has practiced tai chi for 20 years, as a member of the second generation of the Chen-style tai chi master Wang Shi'an.

\section{Acknowledgement}

This article is an achievement of the sub-project of the Art Research Project supported by National Social Science Foundation, "Research on the Training Model of Artistic Talents" [Project Number: 17ZD08].

\section{References}

Encyclopedia of China: Volumes of music and dance. (1989). Beijing: Encyclopedia Press of China. Guriyev, Lipkovkay. (1983). Stage speed and rhythm. Beijing: Chinese Drama Publishing House. Lao Zi. (2008).Dao De Jing, Translated by Ju Yanan, Hollywood: Harvard Square Publishing

Lin, Yutang. (2012). Biography of Su Dongpo , Zhang Zhenyu, Trans.). Changshsa: Hunan Literature and Art Publishing House.

Linklater, K. (2006). Freeing the natural voice: Imagery and art in the practice of voice and language. London: Nick Hern.

Xu Yuanchong. (2007). English Translation of Three Hundred Poems of Tang Dynasty, Beijing: China's Translation Publishing Company

Xu Zhimo. (1983). Poems of Xu Zhimo, Edited by Gu Yongli, Hangzhou: Zhejiang Publishing Company

Zhang, Gengguang.(1991). Translation of Zhuang Zi, Guiyang:Guiyang People Publishing Company, Heng Ta 